\title{
Analytical investigation of the feasibility of sacrificial microchannel sealing for Chip- Scale Atomic Magnetometers
}

\author{
AUTHOR(S): \\ Tsujimoto, Kazuya; Hirai, Yoshikazu; Sugano, Koji; \\ Tsuchiya, Toshiyuki; Tabata, Osamu
}

\section{CITATION:}

Tsujimoto, Kazuya .... [et al]. Analytical investigation of the feasibility of sacrificial microchannel sealing for Chip-Scale Atomic Magneto meters. Microsystem Technologies 2014, 20(3): 357-365

\section{ISSUE DATE:}

2014-03

URL:

http://hdl.handle.net/2433/199891

\section{RIGHT:}

The final publication is available at Springer via http://dx.doi.org/10.1007/s00542-0131895-8.; This is not the published version. Please cite only the published version.; この論 文は出版社版でありません。引用の際には出版社版をご確認ご利用ください。 


\section{Introduction}

Atomic MEMS (Micro Electro Mechanical Systems) are emerging multidisciplinary applications, which include not only CSACs (Chip Scale Atomic Clocks) but also CSAMs (Chip Scale Atomic Magnetometers) with high sensitivity and low cost, which are applicable for wide range of applications such as geophysical exploration, navigation and biomedical imaging (Knappe et al. 2004, Sander et al. 2012). One of critical parts of a CSAM is a microfabricated alkali metal vapor cell (vapor cell), which contains alkali metal vapor such as $\mathrm{K}$ or $\mathrm{Rb}$ and inert gases such as $\mathrm{He}$ or $\mathrm{N}_{2}$. Since performances of a CSAM including sensitivity and lifetime strongly depends on atmosphere inside a vapor cell such as density of the alkali metal vapor and pressure of the inert gases, sealing techniques enabling to control atmosphere inside a vapor cell are crucial (Allred et al. 2002). Although various microfabrication techniques for a vapor cell have been demonstrated, there are still many challenges in establishment of a safe and easy microfabrication technique enabling to control atmosphere inside a vapor cell for accelerating industrialization of CSAMs. These conventional microfabrication techniques can be categorized into two approaches by encapsulation of alkali metal; one is the approach of injection and another is the approach of on-site production as schematically summarized in Fig.1. In the approach of injection, pure alkali metal that is produced outside a vapor cell is injected into it utilizing a pipette (Eklund et al. 2008), microchannels (Lee et al. 2004), or wax containers (Radhakrishnan et al.2005). A main advantage of the approach of injection is high controllability of atmosphere inside a vapor cell, since the vapor cell is finally sealed after elimination of unwanted gases such as air and water, and filling of inert gases. A main drawback of the approach is difficult manipulation of alkali metal that is easy to be oxidized in the atmosphere. On the other hand, in the approach of on-site production, alkali metal is produced inside a sealed vapor cell by thermally activated chemical reaction of solid sources (Liew et al. 2004). A main advantage of the approach of on-site production is easy manipulation of the unreactive sources in the atmosphere. A main drawback of the approach is trapped unwanted gases such as air, water and gaseous byproducts of the chemical reaction, which oxidize the produced alkali metal or degrade the controllability of the atmosphere inside the vapor cell. Recently a novel microfabrication technique utilizing localized heating of the AMD (Alkali Metal Dispenser(ref)) by a laser, which can be seen as one of approaches of the on-site production, has been proposed for CSACs (Knapkiewicza et al. 2010). A reductant in the AMD acts as a getter that absorbs the unwanted gases even after a vapor cell is sealed. However, a chromium (VI) compound of alkali metal that is one of ingredients of the AMD is a carcinogenic substance and use of the compound is strictly restricted in many countries (ELV 2000, RoHS 2002). In addition, the reductant is an aluminum alloy, which can become noise source of CSAMs (Lee et al. 2008). Therefore the microfabrication technique that satisfies all the followings are required.

- $\quad$ Use of safe and stable solid sources to produce alkali metal onsite a vapor cell

- Elimination of the unwanted gases and byproducts not to degrade the controllability of a vapor cell

- $\quad$ Hermetic sealing to maintain realized optimum atmosphere inside a vapor cell

Thus the authors have proposed a novel microfabrication technique utilizing microchannels at a bonded interface as gas feedthrough for evacuating unwanted gases and filling inert gases into a vapor cell, followed by sealing the microchannels by reflow of glass frit as shown in Fig. 2. Glass frit has been selected as the sealing material since its superior hermeticity $\left(3.4 \times 10^{-17} \mathrm{~Pa} \cdot \mathrm{m}^{3} / \mathrm{s}\right.$ has been verified (Sato et al. 2004)) can keep realized 
atmosphere and its electric insulation assures no negative influence on performance as CSAMs amaong various sealing materials that have been conventionally used in MEMS packaging (Knechtel 2005). From the viewpoint of fabrication, the microchannels can be formed as grooves on a substrate to be bonded or tunnels embedded in glass frit layer. For example, the microchannels on a silicon substrate (silicon microchannels) can be formed by photolithography and etching. The micochannels embedded in glass frit layer (embedded microchannels) can be formed by lost photoresist technique (Hirai et al. 2009). Several cross sectional shapes are formable using wet or dry etching in case of the silicon microchannels. Moving-mask UV lithography can be adopted to pattern the sacrificial layer with desired cross sectional shapes in case of the embedded microchannels (Hirai et al. 2007). The purpose of this paper is to investigate applicability of the two formable microchannels and the formable cross sectional shapes from viewpoint of sealing by clarifying characteristics of glass frit reflow. First of all, minimum cross sectional size of the microchannel for modeling the microchannels to be analyzed is required to be estimated, by employing flow conductance model as described in Chapter 2. Then reflow of glass frit into the two types of microchannels with four different cross sectional shapes were simulated using FEM software as described in Chapter 3.

\section{Estimation of required cross-sectional size of microchannels}

To estimate minimum cross-sectional size of the microchannels that fulfill the function as feedthrough of gases, evacuation time into low vacuum (Fig. 2 Step 3), degree of vacuum during degassing (Fig. 2 Step 3), and filling time of inert gases (Fig.2 step 4) were calculated based on flow conductance model. It is desirable to be within approximately an hour from practical viewpoint to evacuate into low vacuum and fill inert gases and degree of vacuum inside a vapor cell during degassing is desirable to be lower than $10^{-5} \mathrm{~Pa}$ to eliminate the unwanted gases.

In the estimations, it was assumed that a cross-sectional shape of the microchannel was square, volume of the vapor cell was $10^{3} \mathrm{~mm}^{3}$, length of each microchannel was $1 \mathrm{~mm}$ and the microchannels were arrayed as indicated in Fig. 3. Inert gases are filled into the vapor cell through the microchannels by surrounding the vapor cell with gases, which is called "bombing” (Goswami et al. 2008). In the estimation, filling pressure was an absolute pressure of 0.25 MPa, and bombing pressure was an absolute pressure of 0.3 MPa. Methods to calculate evacuation time, degree of vacuum, and filling time are explained as below;

\subsection{Estimation method of evacuation time}

During evacuation of a vapor cell from atmospheric pressure $\left(10^{5} \mathrm{~Pa}\right)$ to low vacuum $\left(10^{-2} \mathrm{~Pa}\right)$, viscous flow model can be used. The evacuation time $\Delta t_{e}$ from $P_{e 1}$ to $P_{e 2}$ is represented as an equation as below (Ulvac, Inc. ed. 2002):

$$
\Delta t_{e}=2.3 \frac{V}{S_{e}} \log _{10} \frac{P_{e 1}}{P_{e 2}}
$$

where $V$ is the cavity volume, $S_{e}$ is the pumping speed, which is represented as an equation as below (Ulvac, Inc. ed. 2002): 


$$
\frac{1}{S_{e}}=\frac{1}{S_{v}}+\frac{1}{C_{\text {sun } \_}},
$$

where $S_{v}$ is pumping speed of a vacuum system including a vacuum pump and vacuum plumbing. $1 / S_{v}$ is negligible because the flow conductance of the vacuum system is much larger than that of the microchannels, $C_{\text {sum_e }}$ is the total flow conductance of arrayed microchannels in viscous flow, which is given by;

$$
C_{s w m_{-} e}=N C_{m_{-} e},
$$

where $N$ is number of the arrayed microchannels, which is indicated in Table 1 . Flow conductance of a microchannel at $25^{\circ} \mathrm{C}$ in viscous flow region $C_{m_{-} e}$ is given by (Ulvac, Inc. ed. 2002):

$$
C_{m_{-\varepsilon}}=1988 \frac{a^{2} b^{2}}{l} \frac{P_{e 1}+P_{e 2}}{2} K_{\varepsilon} .
$$

where $a, b, l$ are geometric parameters indicated in Fig. 3. $K_{e}$ is a coefficient given by aspect ratio of cross sectional shape and it is 1 in case of square in viscous flow region (Ulvac, Inc. ed.).

\subsection{Estimation method of degree of vacuum}

After evacuation into low vacuum, the vapor cell is evacuated into ultra high vacuum using a main pump for degassing. In ultra high vacuum, molecular flow model can be used. Degree of vacuum $P_{u}$ is determined by outgassing rate $Q$ and pumping speed $S_{u}$ (Ulvac, Inc. ed. 2002):

$$
P_{u}=\frac{Q}{S_{u}}
$$

In this region, $S_{u}$ can be seen as total flow conductance of the microchannels $C_{\text {sum } \_u}$ by the same reason as in viscous flow, which can be calculated using an equation as below:

$$
C_{s w n_{-} u}=N C_{m_{-} u},
$$

where $C_{m_{-} u}$ is flow conductance of a microchannel in molecular flow region, which is given by (Ulvac, Inc. ed. 2002):

$$
C_{m_{-} u}=309 \frac{a^{2} b^{2}}{(a+b) l} K_{u} .
$$

$K_{u}$ is coefficient given by aspect ratio and it is 1.115 in molecular flow region (Ulvac, Inc. ed.). Outgassing rate $Q$ can be seen as the highest outgassing rate of the parts after enough degassing. Outgassing rate per unit area from borosilicate glass, which is assumed to be used as optical window in our proposed vapor cell, can be decreased at $10^{-8} \mathrm{~Pa} \cdot \mathrm{m}^{3} / \mathrm{s} \cdot \mathrm{m}^{2}$ after 1day of degassing (Ulvac, Inc. ed. 2002). When surface area of the glass parts is $10^{-4} \mathrm{~m}^{2}$, the outgassing rate is on the order of $10^{-12} \mathrm{~Pa} \cdot \mathrm{m}^{3} / \mathrm{s}$. Even though outgassing rate per unit area of boron glass, which is the main component of the glass frit, is unknown, outgassing rate per unit area of glass is generally on the order of $10^{-7}-10^{-9} \mathrm{~Pa} \cdot \mathrm{m}^{3} / \mathrm{s} \cdot \mathrm{m}^{2}$ (Ulvac, Inc. ed. 2002) and it is assumed to be $10^{-7} \mathrm{~Pa} \cdot \mathrm{m}^{3} / \mathrm{s} \cdot \mathrm{m}^{2}$ for the worst case in the estimation. Since radius of a glass frit particle is $300 \mathrm{~nm}$ and therefore the glass frit sealing layer as indicated in Fig. 3 consists of approximately $10^{8} \mathrm{~s}$ of glass frit particles, total surface area of the glass frit is $10^{-4} \mathrm{~m}^{2}$. Outgassing rate from the glass frit is on the order of $10^{-11} \mathrm{~Pa} \cdot \mathrm{m}^{3} / \mathrm{s}$, multiplying the outgassing rate per unit area $10^{-7} \mathrm{~Pa} \cdot \mathrm{m}^{3} / \mathrm{s} \cdot \mathrm{m}^{2}$ and the total surface area of the glass frit $10^{-4} \mathrm{~m}^{2}$. Outgassing from silicon is negligible (Ulvac, Inc. ed.). During production of alkali vapor, outgassing rate from the heated sources was measured as $10^{-}$ ${ }^{9} \mathrm{~Pa} \cdot \mathrm{m}^{3} / \mathrm{s}$ in our preliminary experiment. Thus, the outgassing rate $Q$ is on the order of $10^{-9} \mathrm{~Pa} \cdot \mathrm{m}^{3} / \mathrm{s}$, which is the highest outgassing rate from heated sources. 


\subsection{Estimation method of filling time}

The filling process is also divided into molecular and viscous flow region. Required time $\Delta t_{f_{-} u}$ to fill the inert gas in molecular flow region (from lower than high vacuum to $10^{2} \mathrm{~Pa}$ ) is calculated by an equation as below (Ulvac, Inc. ed. 2002):

$$
\Delta t_{f_{-} u}=-\frac{V P_{0}}{L} \ln \left(1-\frac{10^{2}}{P_{b}}\right),
$$

$P_{0}$ is atmospheric pressure. $P_{b}$ is bombing pressure. $L$ is standard leak rate. In this region, microchannels can be assumed to be a leak pass. The standard leak rate of microchannels is calculated using an equation as below (Davy 1975):

$$
L=\Delta P C_{H e_{-} u},
$$

$\Delta P$ is pressure difference. $C_{H e \_u}$ is flow conductance in molecular flow region at $25^{\circ} \mathrm{C}$ of helium, which is represented as (Ulvac, Inc. ed. 2002);

$$
C_{H e_{-} u}=C_{a i r_{-} u} \sqrt{\frac{M_{a \dot{ }} T}{293.15 M_{H e}}},
$$

$C_{a i r \_u}$ is equal to $C_{\text {sum_u }}$, which is given by the equation (7). $\mathrm{T}$ is temperature. $\mathrm{M}_{\mathrm{air}}$ and $\mathrm{M}_{\mathrm{He}}$ are molecular weight of air and helium, respectively. Required time $\Delta t_{f_{-} \text {vis }}$ to fill the inert gas in viscous flow region from pressure $P_{\text {vis_1 }}$ to $P_{\text {vis_2 }}$ is calculated by an equation as below (Ulvac, Inc. ed. 2002).

$$
\Delta t_{f_{-} \text {w } s}=\frac{V\left(P_{\mathrm{vis} \_}-P_{\mathrm{vis} \_1}\right)}{C_{H e_{-} \text {us }}\left(P_{b}-P_{v i s_{-} 1}\right)},
$$

$C_{H e \_v i s}$ is flow conductance of microchannel in viscous flow region at $25^{\circ} \mathrm{C}$ of helium of microchannel with a rectangle cross-sectional shape (Ulvac, Inc. ed. 2002):

$$
C_{H e_{-} \text {is }}=\frac{\eta_{\text {air }}}{\eta_{H e}} C_{\text {air_uss }},
$$

$C_{a i r_{-} v i s}$ is equal to $C_{\text {sum_e }}$, which is given by the equation (3). $\eta_{a i r}$ and $\eta_{H e}$ are coefficients of viscosity of air and helium, respectively.

Fig. 4 (a), (b), (c) indicate the calculated results of dependency of evacuation time into low vacuum, degree of vacuum, and filling time of buffer gases on cross-sectional size, respectively. The authors concluded that cross-sectional size of the microchannel is desirable to be more than $10 \mu \mathrm{m}$ in order to evacuate into low vacuum in less than 1 hour.

\section{FEM simulation of microchannel sealing by glass frit reflow}

\subsection{Simulated model}

Glass frit reflow of the two types of microchannels with four different cross-sectional shapes as summarized in Table 2 were simulated to investigate applicability of them. The cross-sectional size was designed based on the conclusion of chapter 2. Cross-sectional area was equal between the calculated microchannels. The melting glass frit is assumed to be purely viscous and incompressible. Simplified conservation equations can be expressed as; 


$$
\begin{gathered}
\nabla \cdot \mathbf{u}=\mathbf{0}, \\
\frac{\partial \mathbf{u}}{\partial t}+(\mathbf{u} \cdot \nabla) \mathbf{u}=-\frac{1}{\rho} \nabla p+v \Delta \mathbf{u}+\mathbf{f} .
\end{gathered}
$$

where $p$ is pressure, $\rho$ is density, $v$ is kinematic viscosity, $\mathbf{u}$ is a velocity vector, and $\mathbf{f}$ is external force vector.

Simulated models were constructed by slice of microchannels and meshed by hexahedron elements. Mesh size was 300-500nm. On the assumption that microchannels are long enough (can be seen as finite) against the scale of width and height (depth), Symmetric boundary conditions were given on two cross-sectional surfaces of the sliced microchannels. The geometry and the boundary conditions are shown in Fig. 5 and were implemented using PLANETS (CYBERNET SYSTEMS co., Ltd.), which is a commercially available FEM software for polymer molding process. Parameters of glass frit used in the simulation are summarized in Table 3.

\subsection{Validation of simulation using flow button method}

To validate the simulation of glass frit reflow using plastic molding FEM, a simulated result of shape change and an experimented one by flow button method were compared. The flow button method is evaluation method of flowablity of reflowed glass frit by observation of shape change after reflow of a press-molded pillar made of glass frit. The authors adopted the method for this validation since real-time observation of the microchannel sealing by glass frit reflow is difficult. Even though the driving force of glass frit reflow in the flow button method is gravity instead of applied pressure that is driving force in the proposed microchannel sealing, the same governing equation can be used in the both cases.

Reflow of a glass frit pillar was carried out in a furnace. Comparison of simulated and experimented pillars is shown in Fig. 6. Simulated shape change showed same tendency as experimentally reflowed glass frit pillar. This result roughly verified applicability of FEM on investigation of characteristics of glass frit reflow simulation.

\subsection{Results}

Simulation of glass frit reflow of the two types of microchannels with four different cross-sectional shapes as summarized in Table 2 was carried out. Captured images of the simulated results are shown in Fig. 7.

In order to discuss what kind of formable microchannels is applicable, difference in behavior of glass frit reflow among different cross sectional shapes was clarified by evaluation of the simulated results in respect to filling rate. The filling rate was defined by ratio of filled sectional area to whole sectional area of a microchannel. Comparison of change of filling rates with time is indicated in Fig. 8. From comparison of the silicon microchannels (A, C, E, G) with the embedded microchannels (B, D, F, H), filling rate of the embedded microchannels were higher than the silicon microchannels as a whole. The silicon microchannels with different cross sectional shapes showed more remarkable difference in filling rate than the embedded microchannels. In the silicon microchannels, the narrower the microchannel is, the lower its filling rate became. Comparing the silicon microchannels (A, E, G), whose aperture were same, the rectangular microchannel (E) with aspect ratio of 0.35 was the most easily reflowed. The microchannel which becomes narrower as it goes deep (A, G) showed slower increase of filling rate than the rectangular microchannel. Especially the V-shaped microchannel (A) showed much slower increase of filling rate around the corner than the others. Comparing the embedded 
microchannels (B, F, H), whose aperture are same, the rectangular microchannel (F) with aspect ratio of 0.35 is the most easily reflowed. Comparing the microchannels (B, D), whose height are same, the rectangular microchannel (D) with aspect ratio of 1.4 is the most easily reflowed. Although the embedded rectangular microchannel (F) with aspect ratio of 0.35 was anticipated to show faster filling than that of the rectangular microchannel (D) with aspect ratio of 1.4 since the microchannel (F) is shorter, their filling rate showed almost same tendency.

\subsection{Discussions}

In order to discuss the difference among different cross sectional shapes and investigate applicability of the two microchannels, characteristics of glass frit reflow was clarified by observing vectors of velocity of reflowed glass frit as indicated in Fig. 9. Glass frit in the silicon microchannels receives larger flow resistance than that in the embedded microchannels, since glass frit flows into grooves along the wall of the microchannels and parallel component of the vector of velocity is larger than vertical one. Therefore, difference in cross sectional shape influences on filling rate. The narrower the silicon microchannels become, the larger flow resistance glass frit receives, since the fastest part of glass frit in the narrower microchannel flows more closely to the wall. Thus to fill corners perfectly, glass frit receive very large flow resistance. This could lead to risk of defects of sealing.

On the other hand, glass frit in the embedded microchannels flows against the wall and component of the vector of velocity that is vertical to the wall is larger than that is parallel to the wall. However, the embedded microchannel also has parallel flow against the wall and thus distance to flow near the wall has influence on filling rate in the silicon microchannel, since glass frit that exists on an interval between microchannels is extruded in parallel. Thus both the distance and height influence on filling rate instead of the cross sectional shape, since parallel flow that is away from the wall does not receive large flow resistance enough to contribute to change in filling rate.

According to the revealed characteristics of glass frit reflow, flow resistance is the most important factor, which influences on behavior of glass frit reflow. Followings are concluded;

- $\quad$ The embedded microchannels are more applicable to the proposed technique than the silicon microchannels.

- When adopting the silicon microchannels by restriction of fabrication, microchannel that has corners with acute angle is not recommended

\section{Conclusions}

The novel microfabrication technique utilizing microchannels at the bonded interface as gas feedthrough, followed by sealing the microchannels by reflow of glass frit has been proposed for CSAMs. Glass frit reflow of the silicon microchannel and the embedded microchannel with four cross-sectional shapes were simulated using FEM software under cross sectional size to perform the function as the feedthrough of gases to investigate applicability of them. The simulated results clarified dependence of filling rate on the type of microchannel and cross sectional shapes. Difference of flow resistance between microchannels was found to be important factor that influence on filling rate. Based on the discussion, the authors concluded that embedded microchannel is 
more applicable on the proposed sealing concept. Experimental verification of hermetic sealing by the proposed technique and fabrication of a vapor cell and demonstration as a CSAM will be future works.

\section{Acknowledgement}

This work is partly supported by the Innovative Techno-Hub for Integrated Medical Bio-imaging of the Project for Developing Innovation Systems, from the Ministry of Education, Culture, Sports, Science and Technology (MEXT), Japan.

\section{References}

Allred J. C., Lyman R. N., Kornack T. W., Romalis M. V., "High-sensitive Atomic Magnetometer Unaffected by Spin-Exchange Relaxation”, Physical Review Letters, Vol. 89, No. 13, 130801, 2002.

Davy J.Gordon, “Calculation for Leak Rates of Hermetic Packages”, IEEE trannsactions on parts, hybrids, and packaging, pp.178-189, 1975.

Directive 2000/53/EC OF THE EUROPEAN PARLIAMENT AND OF THE COUNCIL of 18 September 2000 on end-of life vehicles

DIRECTIVE 2002/95/EC OF THE EUROPEAN PARLIAMENT AND OF THE COUNCIL of 27 January 2003 on the restriction of the use of certain hazardous substances in electrical and electronic equipment

Eklund E. J., Shkel A. M., Knappe S., Donley E., Kitching J., “Glass-blown Spherical Microcells for Chip-scale Atomic Devices”, Sensors and Actuators, A, vol. 143, pp. 175-180, 2008.

Goswami A, Han B, "On the applicability of MIL-Spec-based helium fine leak test to packages with sub-micro liter cavity volumes”, Microelectronics Reliability, Vol. 48, pp.1815-1821, 2008.

Hirai Y., Yoshimune H., Tsujimoto K., Sugano K., Tsuchiya T., Tabata O. "Microchannel Embedded in GlassFrit Layer Bonding for Gas-Filled Sealed Cavity”, , in Proc. of HARMST2009, pp. 153-154, 2009.

Hirai Y., Sugano K., Tsuchiya T., Tabata O., "Moving Mask UV Lithography for Three-dimensional Structuring”, Journal of Micromechanics and Microengineering, Vol.17, pp.199-206, 2007.

Knappe S., Shah V., Schwindt P. D., Hollberg L., Kitching J., “A Microfabricated Atomic Clock”, Applied Physics Letters, Vol. 85, No. 9, pp. 1460-1462, 2004.

Knapkiewicza P., Dziubana J., Walczaka R., Maurib L., Dziubanc P., Goreckic C., "MEMS Caesium Vapour Cell for European Micro-Atomic-Clock”, in Proc. of Eurosensors XXIV, pp. 721-724, 2010. 
Lee C. H., Radhakrishnan S., Lal A., Szekely C., McClelland T. A., Pisano A. P., “A Batch Fabricated Rubidium-Vapor Resonance Cell For Chip-Scale Atomic Clocks”, in Proc. of Hilton Head 2004, pp. 23-26, 2004.

Lee S. K., Romalis M., "Calculation of Magnetic Field Noise from High-permeability Magnetic Shields and Conducting Objects with Simple Geometry”, Journal of Applied Physics, Vol. 103, 084904, 2008.

Liew L., Knappe S., Moreland J., Robinson H., Hollberg L., Kitching J., “Micromachined Alkali Atom Vapor Cells for Chip-scale Atomic Clocks”, in Proc. of MEMS2004, pp.113-116. 2004.

Liew L., Knappe S., Moreland J., Robinson H., Hollberg L., Kitching J., “Microfabricated Alkali Atom Vapor Cells”, Applied Physics Letters, vol. 84, No. 14, pp. 2694-2696, 2004.

Radhakrishnan S., Lal A., “Alkali Metal-wax Micropackets for Chip-scale Atomic Clocks”, in Proc. of Transducers2005, pp.23-26, 2005.

Sander T. H., Preusser J., Mhaskar R., Kitching J., Trahms L., Knappe S., “Magnetoencephalography with a Chip-Scale Atomic Magnetometer”, Biomedical Optics Express, Vol. 3, No. 5, pp. 981-990, 2012.

Sato S., "RF MEMS Packaging Technology(<Special Articles>MEMS Packaging for Commercialization)”, Journal of Japan Institute of Electronics Packaging, vol. 7, no. 4, pp. 299-302, 2004.

Ulvac, Inc. ed., “Sinkuu Handbook (Handbook of Vacuum)”, pp. 40-59, Tokyo: Ohmsha, 2002. 


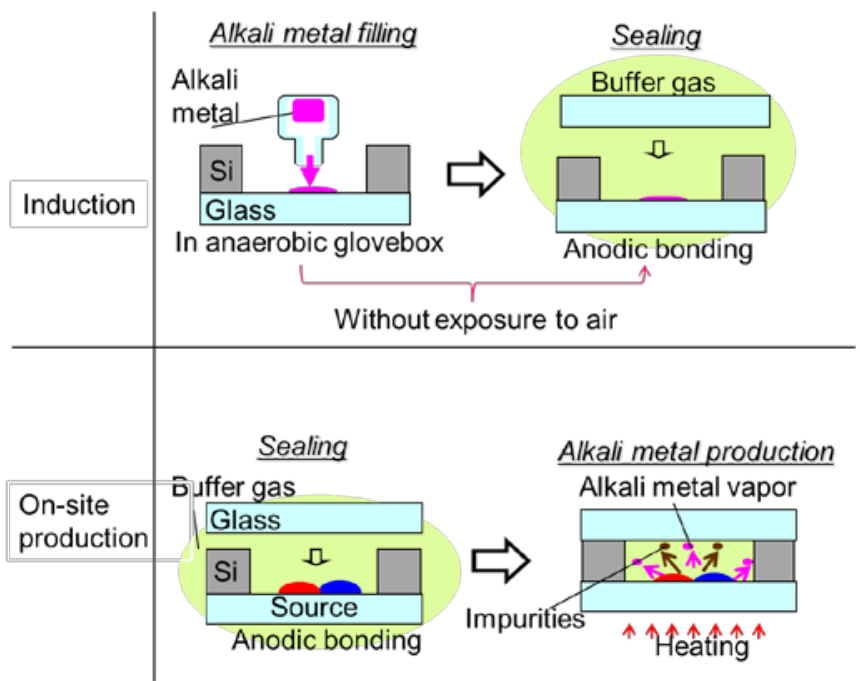

Figure 1. Schematic images of categorized conventional fabrication techniques.

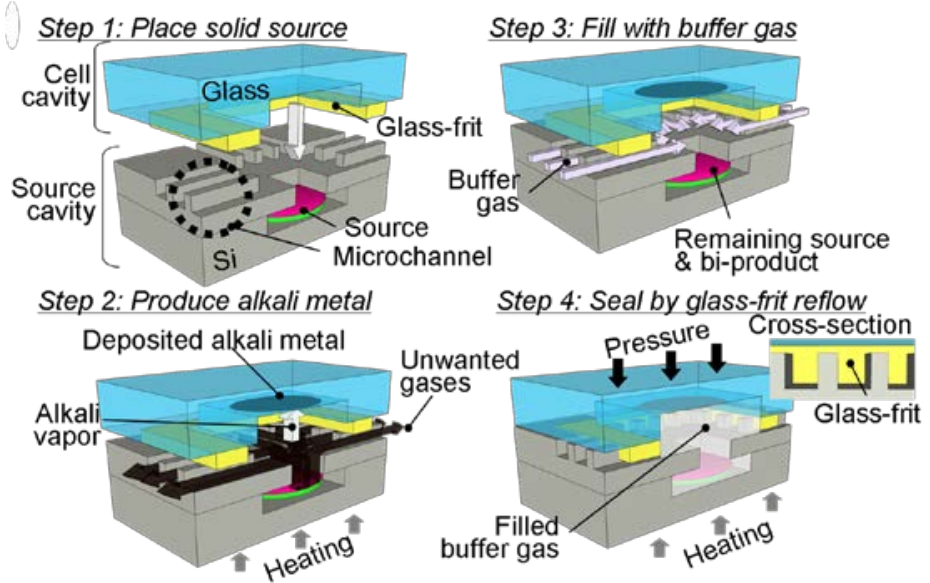

Figure 2. Schematic image of sacrificial microchannel sealing technique.

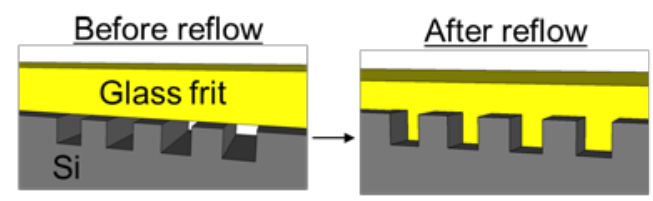

(a) Silicon microchannel

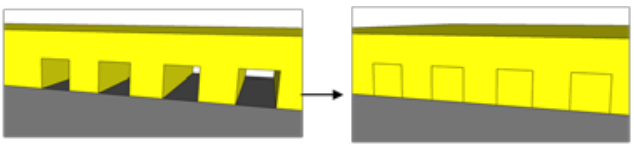

(b) Embedded microchannel

Figure 3. Schematic image of two types of microchannels. (a) Silicon microchannel, (b) Embedded microchannel. 


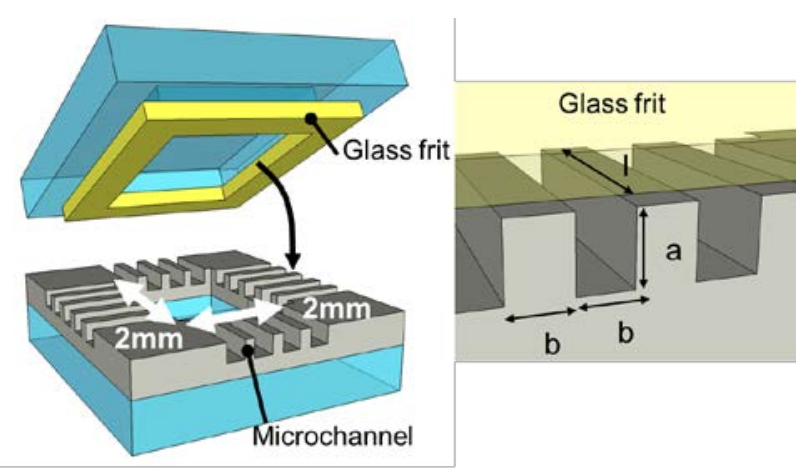

Figure 4. Geometric parameters of assumed vapor cell.

(a)

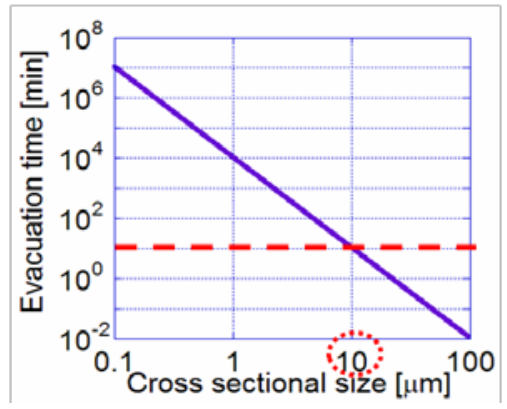

(b)

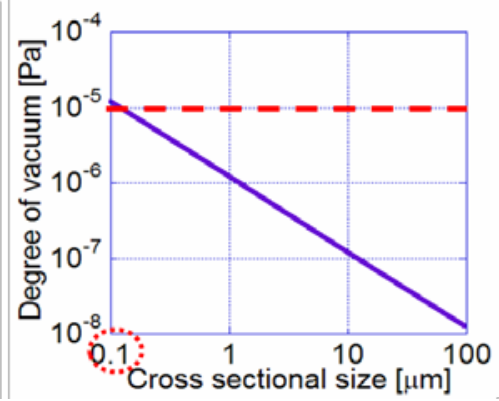

(c)

Figure 5. (a) Evacuation time, (b) Ultimate vacuum, (c) Time for filling buffer gases.
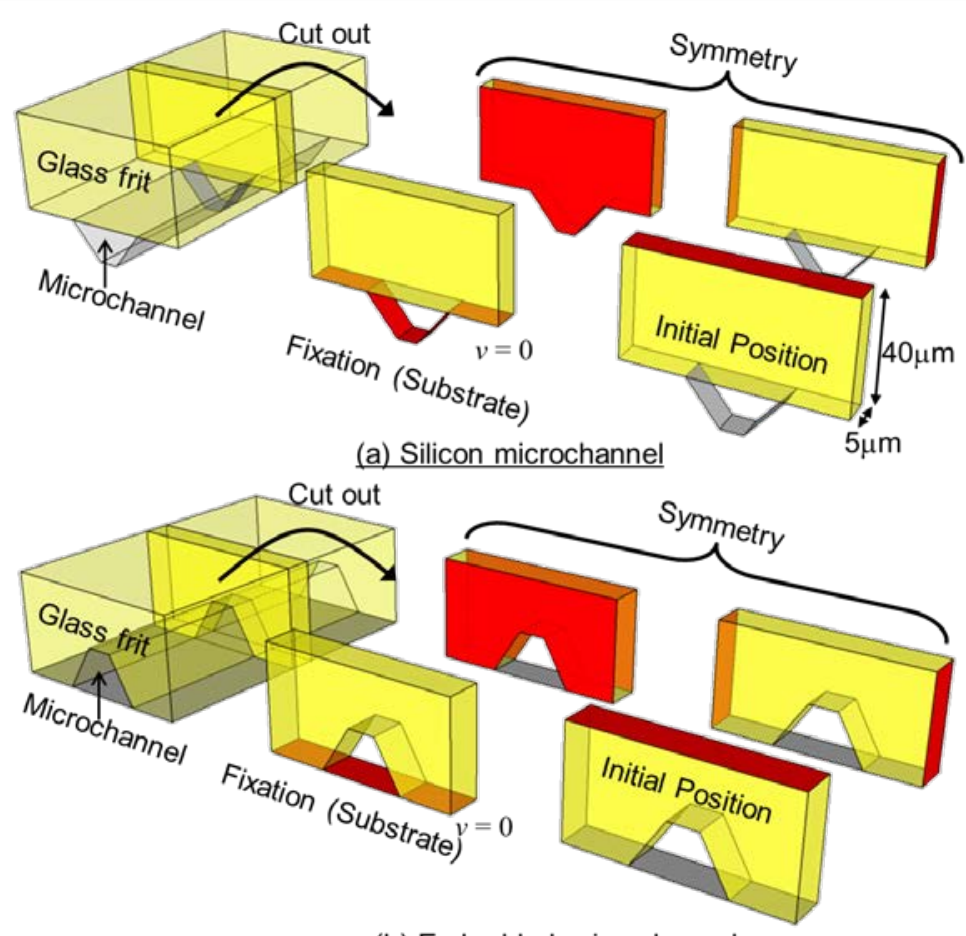

(b) Embedded microchannel

Figure 6. Simulated models and boundary conditions of (a) Silicon microchannels, (b) Embedded microchannels. 


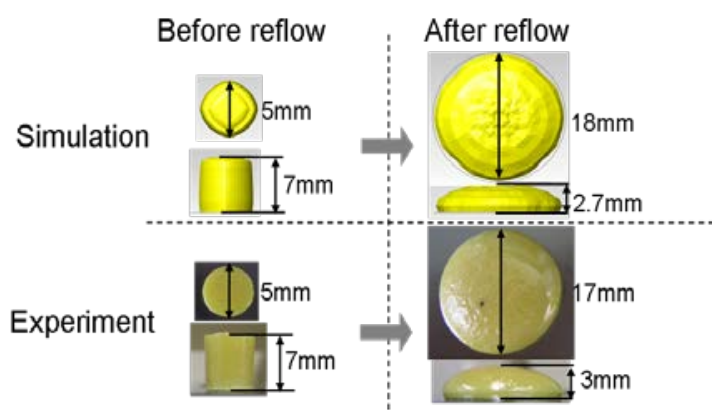

Figure 7. Comparison between simulation and experiment of flow button method.

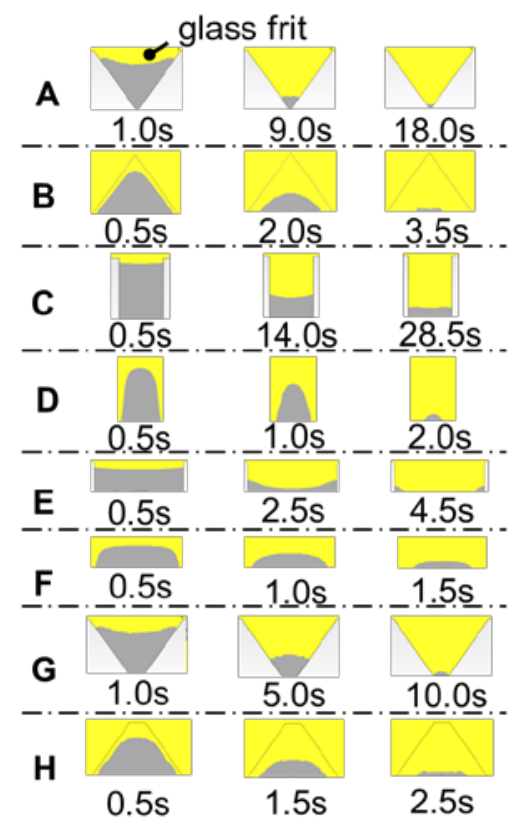

Figure 8. Calculated filling rates of various cross-sectional shapes. 


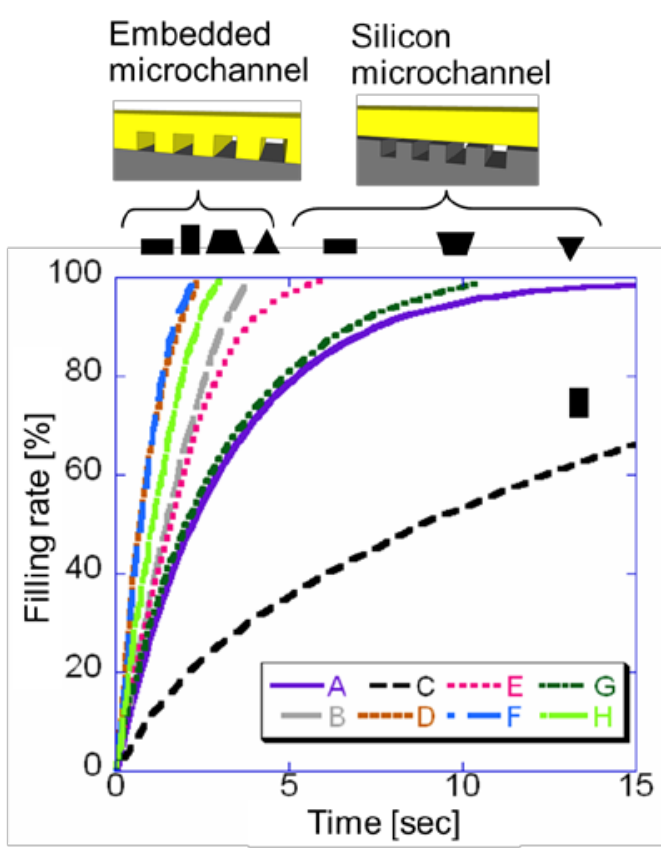

Figure 9. Calculated filling rates of various cross-sectional shapes.

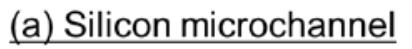

(b) Embedded channel
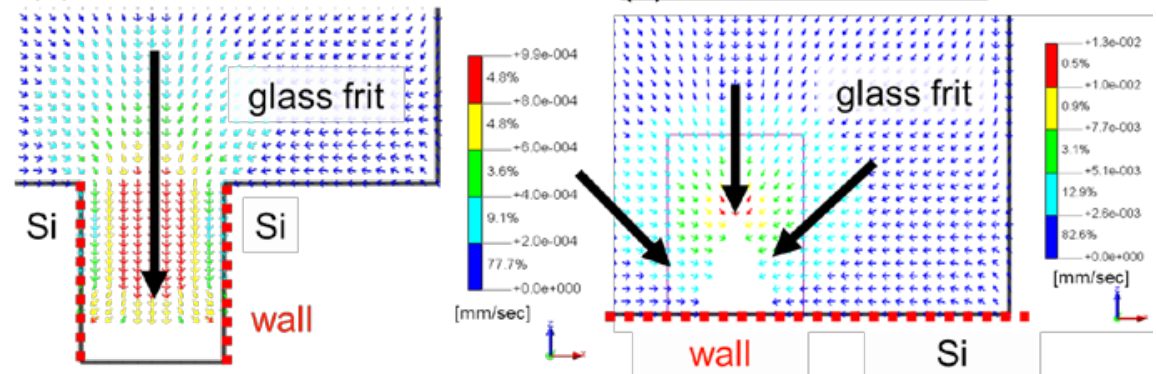

Figure 10. Fig.10. Flow vectors of reflowed glass frit of (a) Silicon microchannel, (b) Embedded microchannel.

Table 1 Number of arrayed microchannels.

\begin{tabular}{c|c}
\hline Cross sectional size & Number of microchannels \\
\hline 0.1 & 40000 \\
\hline 1 & 4000 \\
\hline 10 & 400 \\
\hline 100 & 40 \\
\hline
\end{tabular}


Table 2 Simulated cross sectional shapes.

\begin{tabular}{|c|c|c|c|c|}
\hline & Type & Shape & Width $[\mu \mathrm{m}]$ & Depth(Height) $[\mu \mathrm{m}]$ \\
\hline A & silicon & \multirow{2}{*}{ triangle } & 30 & 21 \\
\hline B & embedded & & 30 & 21 \\
\hline C & silicon & \multirow{2}{*}{ rectangle } & 15 & 21 \\
\hline D & embedded & & 15 & 21 \\
\hline $\mathbf{E}$ & silicon & \multirow{2}{*}{ rectangle } & 30 & 10.5 \\
\hline $\mathbf{F}$ & embedded & & 30 & 10.5 \\
\hline \multirow{2}{*}{ G } & \multirow{2}{*}{ silicon } & \multirow{4}{*}{ trapezium } & aperture 30 & \multirow{2}{*}{18} \\
\hline & & & base & \\
\hline \multirow{2}{*}{$\mathbf{H}$} & \multirow{2}{*}{ embedded } & & aperture 30 & \multirow{2}{*}{18} \\
\hline & & & roof 5 & \\
\hline
\end{tabular}

Table 3 Parameters used in simulation.

\begin{tabular}{c|c}
\hline Temperature $\left[{ }^{\circ} \mathrm{C}\right]$ & 480 \\
\hline Surface tension $[\mathrm{dyn} / \mathrm{cm}]$ & $\mathbf{8 0}$ \\
\hline Contact angle $\left[{ }^{\circ}\right]$ & 100 \\
\hline Viscosity $[\mathrm{Pa} \cdot \mathrm{S}]$ & $10^{7}$ \\
\hline Pressure $[\mathrm{kPa}]$ & 150 \\
\hline Time step $[\mathrm{sec}]$ & $\mathbf{0 . 0 5}$ \\
\hline
\end{tabular}

